\title{
Exclusion of a submitral pseudoaneurysm by a conservative transmitral approach - Surgical technique for an unusual disease
}

\author{
Alexandre Sebestyen ${ }^{1}$, Fahd Bennani ${ }^{1}$, Claude Koulekey ${ }^{1}$, and Olivier Chavanon ${ }^{1}$ \\ ${ }^{1}$ University Hospital Centre Grenoble Alpes
}

May 28, 2020

\begin{abstract}
Cardiac pseudoaneurysm is a contained rupture of the cardiac wall. Rarely symptomatic, the risk of death by stroke or rupture is high and suggests surgical treatment. Surgical strategy depends on its anatomical considerations. We reported the case of a submitral pseudoaneurysm. We excluded it by a conservative transmitral approach, without any short- and long-term complication.
\end{abstract}

\section{Introduction}

Cardiac pseudoaneurysm is a contained rupture of the cardiac wall ${ }^{1}$. Although myocardial infarction is the main etiology, traumatic lesions are more commonly described with chest penetrating as the first cause of trauma ${ }^{1}$. Cardiac surgery and, rarely, endovascular procedures are also possible ${ }^{2-5}$. Rarely symptomatic, the risk of stroke or rupture is high and suggests surgical treatment ${ }^{1}$.

We present the anatomical considerations of a rare case of submitral pseudoaneurysm in a young woman. We excluded it by a conservative transmitral approach.

Case

A 28-years-old woman presented with symptoms evocating pulmonary embolism. Thoracic computed tomography angiography disproved it but showed a pseudoaneurysm of the left ventricle. We only noted a prior ablation of accessory pathway for Wolff-Parkinson-White syndrome by retrograde transaortic approach, 12 years ago ${ }^{6}$.

The pseudoaneurysm was 46 millimeters in diameter and partially thrombosed. Its orifice was 12 millimeters in diameter and localized on the lateral face of the left ventricle near the mitral annulus (Figure 1). There was no mitral regurgitation and left ventricular function was preserved. The calcified wall of the pseudoaneurysm was close to the circumflex artery which was posteriorly repressed (Figure 1). There was no extrinsic stenosis of the circumflex artery and the rest of the coronary network was strictly normal. Because this pseudoaneurysm was symptomatic and the risk of rupture was high, intervention was indicated.

By sternotomy and left atriotomy approach, we exposed the mitral valve and found the orifice behind P1 (Figure 2a). We detached the posterior mitral valve from the anterior commissure to the median portion of P2 (Figure $2 \mathrm{~b}$ ). We closed the orifice by suturing a round piece of Dacron patch (HEMASHIELD ${ }^{\mathrm{TM}}-\mathrm{Maquet}$ Germany). We first placed a crown of pledgeted \#4-0 polypropylene U-stitches passed from the inside of the pseudoaneurysm to the ventricular bank around the orifice (Figure 2c). The circular patch of 3 centimeters in diameter, covering almost 1 centimeter around the orifice, was then attached (Figure 2d). Suture was reinforced around with some \#5-0 polypropylene stitches. Finally, we reattached the posterior mitral valve by a run suture like a sliding valve repair (Figure 2e). Time of clamp was 105 minutes. Intraoperative transesophageal echocardiography confirmed the disappearance of any communication between the left ventricle 
and the pseudoaneurysm, without mitral regurgitation. Computed tomography before discharged confirmed the sealing of our repair with total thrombosis of the pseudoaneurysm. The patient discharged from hospital at postoperative day 9. Computed tomography at month 3 and month 15 confirmed the persistence of the sealing (Figure 3). Thereafter, clinical and echocardiographic follow-up was done, stopping irradiation in this young woman. Finally, no events such as recirculation of the pseudoaneurysm, stroke, myocardial infarction, mitral regurgitation or death during follow-up was reported until postoperative month 48.

\section{Discussion}

Cardiac pseudoaneurysms are rare conditions. Rarely symptomatic, their prognosis remains poor with a risk of rupture or stroke of $40-50 \%$ within the first year ${ }^{1}$. Myocardial infarction is the main etiology but post-surgical or -interventional pseudoaneurysms are described ${ }^{1-5}$. Surgical treatment is indicated with mortality of $20-30 \%^{1}$. Percutaneous closure is described but the submitral localization make the procedure complex, increasing the risk of embolization or mitral regurgitation ${ }^{7}$.

Several surgical techniques to treat cardiac pseudoaneurysms are described, depending on its localization and the quality of the perianeurysmal cardiac tissue ${ }^{8,5,9}$. Submitral position, close to the circumflex artery, increased the risk of myocardial infarction or need of coronary bypass on the lateral wall ${ }^{8,10}$. In addition, calcifications on the wall made the suture fragile in this high mechanical stress region, increasing the risk of tear in the left atrioventricular groove ${ }^{5,8}$.

To minimize both theses complications, we decided on an inside exclusion rather than a resection ${ }^{5,8}$. Inside exclusion by left atriotomy was first described by Antunes to treat congenital submitral aneurysm by transatrial approach, incising the floor of the atrium to access inside the aneurysm by its roof ${ }^{11}$. In our case, the circumflex artery was posteriorly repressed between the left atrium and the pseudoaneurysm which were not in-contact, that made this way unsuitable ${ }^{10}$. We decided on a transmitral approach to close the orifice from the inside of the left ventricle. Transmitral approach was first described for post-infarction pseudoaneurysm when the mitral valve was also replaced ${ }^{12}$. In this young woman, we aimed to preserve the mitral valve and took great care on performing a partial detachment-reattachment of the posterior mitral valve. The detachment of the posterior valve, already described by Miura et al., gave access to the orifice just behind the annulus without being bothered by the subvalvular apparatus ${ }^{5}$. We closed the orifice with a patch rather than a direct suture to avoid mitral regurgitation by subvalvular geometry distorsion ${ }^{8}$. Pledgeted U-stitches were performed thick enough but taking care of not to injure the circumflex artery. Finally, although the left atriotomy approach could be performed by video-assisted right minithoracotomy, the coronary risk led us to prefer a sternotomy.

Closure was efficient and total thrombosis was done and permanent without any short- and long-term major events. Unlike post-infarction pseudoaneurysms, traumatic ruptures seem to be well limited and surrounded by firmness myocardial tissue which might explain the durability of our sealing repair.

\section{Conclusion}

Cardiac pseudoaneurysm remains a rare condition. Several surgical techniques are described and each one should be adapted for each case depending on the localization of the pseudoaneurysm. Inside exclusion by left atriotomy and transmitral approach seems a safe option to know for submitral pseudoaneurysms, minimizing the risk of coronary lesion and bleeding.

Figure legends

\section{Figure 1 - Anatomical considerations of the submitral pseudoaneurysm}

Computed tomography (left) and 3D-reconstruction (right) showed the orifice $\left({ }^{*}\right)$ of the pseudoaneurysm (PsA) just behind the mitral annulus (MA). The circumflex artery $(\mathrm{Cx})$ was posteriorly repressed, between the PsA and the left atrium (LA) which were not in-contact. Calcifications were on the PsA wall.

Figure 2 - Surgical exclusion by a conservative transmitral approach 
The orifice $\left(^{*}\right)$ was localized behind P1 (a). First, the posterior mitral valve was partially detached and the Dacron patch was cut according to the size of the orifice (b). Then, pledgeted "U" threads surrounded the edge of the orifice and the Dacron patch was sutured to close it $(c, d)$. Finally, the posterior mitral valve was reattached by a sliding valve repair technique (e)

Figure 3 - Result of surgery

Total thrombosis of the pseudoaneurysm (PsA) confirmed the sealing of our repair $\left(^{*}\right)$.

References

1. Frances C, Romero A, Grady D. Left ventricular pseudoaneurysm. J Am Coll Cardiol. 1998;32(3):557-561.

2. Shirota K, Yano Y, Hayase S, et al. [A case of submitral left ventricular pseudoaneurysm following mitral valve replacement for infective endocarditis].Kyobu Geka. 1998;51(5):410-414.

3. Lanjewar C, Thakkar B, Kerkar P, Khandeparkar J. Submitral left ventricular pseudoaneurysm after mitral valve replacement: early diagnosis and successful repair.Interact Cardiovasc Thorac Surg. 2007;6(4):505-507.

4. Namboodiri N, Dora SK, Thomas B, Misra M. Subannular left ventricular pseudoaneurysm following mitral valve replacement. J Cardiothorac Surg. 2008;3(1):28.

5. Miura T, Yamazaki K, Kihara S, et al. Transatrial repair of submitral left ventricular pseudoaneurysm. Ann Thorac Surg. 2008;85(2):643-645.

6. Auriau J, Jankowski A, Defaye P. Large spherical left ventricular pseudoaneurysm: a very rare long-term complication of ablation of an accessory pathway.EP Eur. 2017;19(10):1680-1680. doi:10.1093/europace/euw259

7. Kumar PV, Alli O, Bjarnason H, Hagler DJ, Sundt TM, Rihal CS. Percutaneous therapeutic approaches to closure of cardiac pseudoaneurysms. Catheter Cardiovasc Interv. 2012;80(4):687-699.

8. Jahangiri M, Sarkar D, Quinton P, Ward DE. Submitral left ventricular pseudoaneurysm.Ann Thorac Surg. 2005;79(3):1031-1032.

9. Prifti E, Bonacchi M, Baboci A, et al. Surgical treatment of post-infarction left ventricular pseudoaneurysm: Case series highlighting various surgical strategies. Ann Med Surg. 2017;16:44-51.

10. Purushotham S, Manohar SRK, Sivasubramaniam S, Neelakandhan KS. Submitral left ventricular aneurysm: The location of the circumflex coronary artery in relation to the aneurysm influences the surgical approach and outcome.J Thorac Cardiovasc Surg. 2005;129(5):1175-1177.

11. Antunes MJ. Submitral left ventricular aneurysms. Correction by a new transatrial approach. J Thorac Cardiovasc Surg. 1987;94(2):241-245.

12. Da Col U, Di Bella I, Ramoni E, Affronti A, Rossi A, Ragni T. Repair of posterior left ventricular aneurysm through transatrial approach. J Card Surg. 2010;25(1):23-25. 

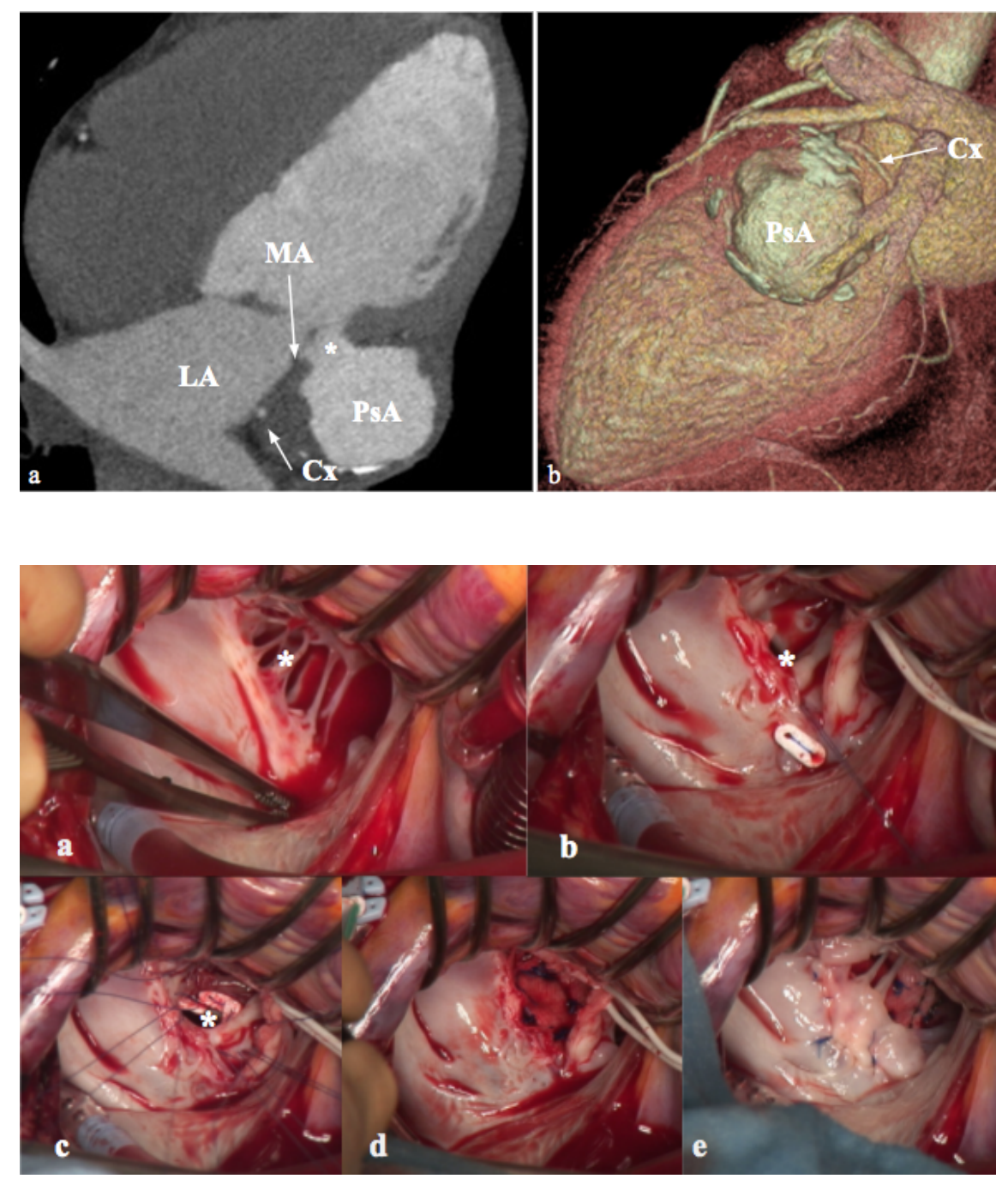


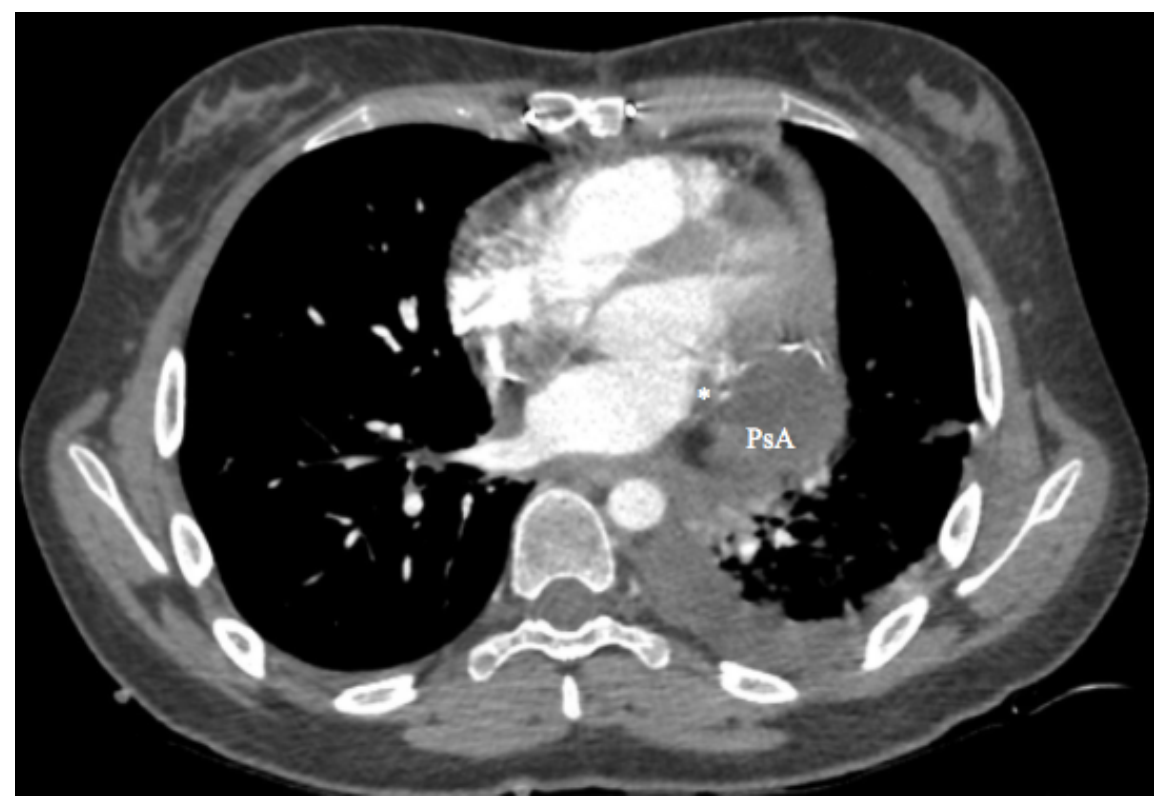

